\title{
Association between diet and cardiovascular disease and overall mortality risk in France and Northern Ireland: the PRIME study
}

\author{
N. McCloskey ${ }^{1}$, M. C. McKinley ${ }^{1}$, D. Arveiler ${ }^{2}$, B. Haas $^{2}$, P. Amouyel ${ }^{3}$, J. Dallongeville ${ }^{3}$, J. Ferrières ${ }^{4}$, \\ V. Bongard ${ }^{4}$, F. Kee ${ }^{1}$, A. Bingham ${ }^{5}$, P. Ducimetière ${ }^{5}$ and J. V. Woodside ${ }^{1}$ \\ ${ }^{1}$ Centre of Public Health, Queen's University Belfast, Belfast, Northern Ireland, ${ }^{2}$ MONICA-Strasbourg, France, ${ }^{3}$ MONICA \\ - Lille, France, ${ }^{4}$ MONICA-Toulouse, France and ${ }^{5}$ INSERM U780, Villejuif, France
}

Traditional dietary assessment has examined nutrients or foods in isolation ${ }^{(1)}$. Such an approach has limitations due to complexities of dietary intake ${ }^{(1)}$. Assessment via dietary patterns has recently emerged as a complementary approach, accounting for synergistic effects of food consumption ${ }^{(2)}$.

The aim of this study was to calculate a priori and a posteriori dietary scores in the PRIME prospective cohort study. PRIME was designed to examine the substantial differences in cardiovascular incidence and mortality between Northern Ireland and France. The PRIME study included dietary data on men aged 50-59 y recruited during 1991-1993 from Belfast, Northern Ireland ( $n=2748)$, and Lille $(n=2627)$, Strasbourg $(n=2611)$, and Toulouse $(n=2610)$ in France. Detailed medical and lifestyle questionnaires were collected at baseline, as was a short, 16-item food frequency questionnaire. Participants were followed-up annually. Over 10 year follow-up, <5\% of men were lost, while $n=654$ men died, and $n=546$ had a cardiovascular disease (CVD) event, and these events were validated by a medical committee.

From the available dietary data, four a priori and four a posteriori dietary scores were calculated. A priori scores included: Fruit and Vegetable (FV) Variety Score, Overall Variety Score, Adapted Mediterranean Diet Score (aMDS), and Daily FV Score. Two French and two Northern Irish a posteriori dietary patterns were created using principal component analysis; these were labeled "F1:Healthy" and "F2:Unhealthy" (French patterns), "B1:Vegetables and Nuts", and "B2:Fruit, Vegetables and Fish" (Belfast patterns) respectively. Cox's Proportional Hazard Regression was used to determine the association between these patterns and risk of overall mortality or CVD incidence; $p$ values were determined using a trend test across four categories of diet score.

After adjustment for country, age, and other biological and lifestyle variables: 4 scores were significantly associated with overall mortality. In the highest fourth, showing greatest adherence to diet pattern: the "B2:Fruit, Vegetable and Fish" Score and "F1:Healthy" Score were associated with reduced overall mortality (HR 0.74 (95\% CI: 0.57-0.96), p ptrend $=0.049$ and HR 0.74 (95\% CI 0.57-0.96), $\mathrm{p}_{\text {trend }}=0.02$ respectively). Daily FV Score and aMDS were also associated with reduced overall mortality (HR 0.75 (95\% CI: $0.57-0.99$ ), $\mathrm{p}_{\text {trend }}=0.03$ and HR 0.73 (95\% CI: 0.50-1.05), $\mathrm{p}_{\text {trend }}=0.049$ respectively). The remaining scores were not associated with overall mortality. Although trends were observed in a similar direction, no dietary patterns were associated with CVD risk in the fully adjusted models.

In conclusion, adhering to healthy dietary patterns is associated with a reduction in all-cause mortality risk of around $25 \%$. Higher intakes of fruit, vegetables and fish may have a protective effect on overall mortality in middle-aged men. The association observed between diet and overall mortality and CVD risk depends on dietary pattern methodology used.

1. Hu FB (2002) Curr Opin Lipidol 13, 3-9.

2. Jinlin F, Binyou W \& Terry C (2007) J Postgrad Med 53, 139-43. 